\title{
History-dependent rheology of a surfactant hexagonal phase
}

\author{
M. L. Sushko, * J. M. Seddon, and R. H. Templer \\ Department of Chemistry, Imperial College, Exhibition Road, London SW7 2AY, United Kingdom \\ (Received 16 August 2001; revised manuscript received 7 November 2001; published 11 February 2002)
}

\begin{abstract}
The time-dependent response of a surfactant hexagonal phase of a sodium dodecyl sulphate/pentanol/ cyclohexane/brine system to stepped strain is investigated. The dynamics of the system is found to be governed by strain- and noise-induced yielding of the domains of the system. The effects of the applied strain magnitude and the ionic strength of the brine on the character of the transitions experienced by the system are reported.
\end{abstract}

DOI: 10.1103/PhysRevE.65.031501

PACS number(s): 83.50.-v, 83.80.Qr

\section{INTRODUCTION}

Many soft materials exhibit history-dependent rheology. Two different types of history-dependent responses have been defined: rheological aging, and a "transient" behavior of a power-law fluid. The recent theory of the rheological behavior of soft glassy materials, the soft glassy rheology (SGR) model, describes both cases. Aging (of the step strain response) is defined in the SGR model $[1,2]$ as the property that a significant part of the stress relaxation takes place on time scales that grow with the age of the system. Contrary to the case of aging systems, in "transient" behavior all significant relaxation processes can essentially be observed on finite time scales. However, in "transient" systems the time scales of the relaxation after the step strain also depend on the age of the system at the time of strain application. Thus, these systems have a short-term memory. Transient behavior has been found in dense emulsions [3], foams [4], and polydomain defect textures in ordered mesophases [5], whose rheology is governed by rearrangements of domains.

The aging phenomenon of pastes [6], flocculated dispersions [7], colloidal glasses [8], and some other systems is well described experimentally and a good agreement with the theory has been reported. However, the historydependent rheology of "transient" systems seems not to have been directly investigated. In this paper we will describe the history-dependent response upon shear of a surfactant hexagonal phase. First we give a brief overview of the experimental data on the rheology of lyotropic hexagonal phases.

Surfactant hexagonal phases have been found to experience transitions to some new stable states of director orientation under steady shear or constant stress [9]. Small angle light scattering studies revealed correlated orientational fluctuations perpendicular to the flow direction at low creep times or at low shear rates, but parallel to the flow direction at long creep times or high shear rates [10]. The correlation of orientational fluctuations, perpendicular to the flow direction, was attributed to log rolling of the cylindrical micelles, predicted theoretically as a possible state of orientation for polymeric liquid crystals under shear [11]. However, rheonuclear magnetic resonance investigations revealed that the only stable state of the director orientation under shear was

\footnotetext{
*Corresponding author.
}

an orientation parallel to the flow [12]. Thus the correlation of orientational fluctuations perpendicular to the flow direction could not be attributed to the log rolling of the micelles, but was suggested to reflect an undulation of the director with a period of several micrometers [13]. It was found that once the system underwent a transition to some new state of the director orientation, it remained in this state even after cessation of shear [14-16].

An important feature of the rheological behavior of a hexagonal phase is the independence of the director orientation on the shear rate in steady shear experiments. In this regime the tilt angle $\vartheta$ of the director, initially aligned along the velocity gradient was found to be given by $\gamma=\arctan \vartheta$, where $\gamma$ is the strain, indicating that the hexagonal phase behaves as a deformable solid [12].

Recently a class of highly swollen surfactant hexagonal phases composed of a mixture of sodium dodecyl sulphate, pentanol, brine, and cyclohexane has been discovered and this has allowed us to investigate the rheology of the hexagonal phase in more detail. The advantage of the system is that the radius of the oil swollen cylinders, $R$, can be continuously changed by an order of magnitude, while the intercylinder distance remains constant, by varying the oil content and the ionic strength of the polar medium [17]. This leads to a dramatic change in the elasticity of the system [18]. The macroscopic elastic modulus was found to scale as $1 / R^{3}$. The results of creep tests reported on these soft systems gives some evidence in favor of the SGR model for systems with transient behavior, revealing, however, that the rheological response of the system is more complicated than that described in the model [18]. It was found experimentally that at long creep times ( $t \geqslant 100 \mathrm{~s}, t$ being the stress-on time) the strain increases as a power law with $t$

$$
\gamma=\zeta t^{\alpha}
$$

Comparing the experimentally obtained strain time dependence having the form of Andrade's law for primary creep in metals [19], with the general equation for creep

$$
\gamma=\sigma J(t),
$$

and by substituting the creep compliance $J(t)$ with the expressions given in the SGR model

$$
(x>2): \quad J\left(t-t_{w}\right) \propto\left(t-t_{w}\right) \quad \text { for linear regime, }
$$




$$
(1<x<2): \quad J\left(t-t_{w}\right) \propto\left(t-t_{w}\right)^{x-1} \quad \text { for transient regime },
$$

one can see that Andrade's law (1) for a polycrystalline system corresponds to expression (4) given for the "transient" regime in the SGR model with the exponent $\alpha$ equal to $x$ -1 [note, $\left(t-t_{w}\right)$ in the SGR model is the measuring time, i.e., time elapsed from the beginning of the measurement and $t_{w}$ is the time of stress application, the waiting time]. The exponent $\alpha$ has been reported to be independent of the history of the sample for a given applied stress so long as a polycrystalline texture was maintained in agreement with Eq. (4) showing no waiting time dependence of the creep compliance. However, the exponent was found to depend on the imposed stress while $x$ is taken to be a constant in the SGR model. According to the experimental results [18], the exponent $\alpha$ increases with the applied stress from 0.62 at $\sigma=1 \mathrm{~Pa}$ up to 0.9 at $\sigma=9 \mathrm{~Pa}$. This corresponds to an increase of $x$ $=\alpha+1$ with applied stress from 1.62 up to 1.9 in the polycrystalline regime. Further increase of the applied stress leads to monodomain formation, and, consequently, to a new rheological behavior of the system. It was found that for large stress, the system reaches a steady state with $\alpha=1$. The same steady state, characterized by a linear variation of $\gamma$ with time, was immediately reached, if the initially polycrystalline system was presheared by the application of a sufficiently large stress [18]. These results are in agreement with the theory, which gives the expression for the creep compliance in the case of $x>2$ different to that for lower $x$, with the compliance becoming linearly dependent on the measuring time [see Eq. (3)].

Thus, this surfactant system seems to be very suitable for a detailed investigation of history-dependent rheology in the transient regime.

\section{EXPERIMENTAL PROCEDURE}

We investigated the quaternary system of sodium dodecyl sulfate (SDS) (BDH Laboratory Suppliers, England, 99\% purity), pentanol, brine, and cyclohexane. Systems with 0.5 and $0.4 M$ brine were used. In both systems the brine/SDS weight ratio was equal to 2.5 while the cyclohexane and pentanol content was 65 and 4 wt. \% of the total weight of the mixture, respectively, for the system with $0.5 \mathrm{M}$ brine, and $61.5 \%$ and $4.7 \%$ for the system with $0.4 M$ brine. At the above compositions the systems are reported to display swollen hexagonal phases at room temperature [17], and we confirmed this by polarizing microscopy.

The rheological measurements were performed in a Couette geometry on a strain-controlled Bohlin VOR rheometer (BRS VOR 7:9, Sweden). The Couette cell consisted of a fixed inner cylinder of $7 \mathrm{~mm}$ radius and a rotating outer cylinder, with a $0.7 \mathrm{~mm}$ sample gap.

In the relaxation tests, a strain $\gamma_{0}$ was applied and the time dependence of the relaxation modulus $G(t)=\sigma(t) / \gamma_{0}$ was measured [here $\sigma(t)$ is the stress]. A series of experiments with applied strains of 20\%, 40\%, 60\%, 80\%, and $100 \%$ were performed. Each of the series of measurements consisted of 19 relaxation experiments lasting $300 \mathrm{~s}$ each

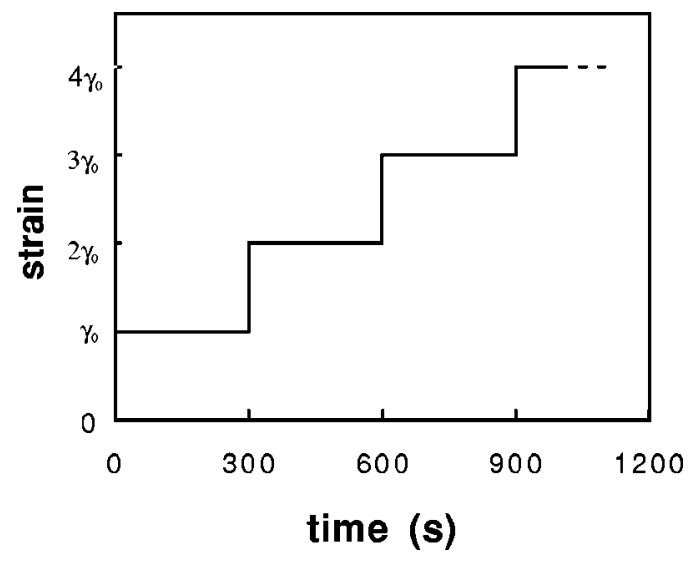

FIG. 1. Time dependence of strain applied to SDS/pentanol/ cyclohexane/brine systems. The values for $\gamma_{0}$ are given in the text.

with a strain rise time of $0.1 \mathrm{~s}$ (Fig. 1). Each successive relaxation measurement in the series started immediately after the previous one was finished. In other words the waiting time $t_{w}$, i.e., the time elapsed from the beginning of the first measurement in the series, had an increment of $300 \mathrm{~s}$ from measurement to measurement. All relaxation experiments were performed at $25 \pm 0.2^{\circ} \mathrm{C}$.

To avoid any preshearing of the sample, it was cooled down to $+2{ }^{\circ} \mathrm{C}$ once loaded in the measuring system (at this temperature the sample is in an isotropic state) and then heated up to $25^{\circ} \mathrm{C}$ and left at this temperature for $4 \mathrm{~h}$ or more before the measurements were performed. This procedure gave reproducible results. All experiments were repeated at least three times.

\section{THEORY: THE SOFT GLASSY RHEOLOGY MODEL}

In the SGR model [1] the macroscopic sample is regarded as a combination of mesoscopic elements. Each element is assigned a local strain $l$, and a corresponding stress $k l$ ( $k$ is an elastic constant), which describes deformations away from some local position of unstressed equilibrium relative to neighboring elements. The local strain of an element is supposed to follow the imposed strain until it reaches its yield value $l_{y}$. At this point the element rearranges to a new configuration, where it is less deformed. Thus, yielding provides a mechanism of stress relaxation, while between yield events the material behaves as an elastic solid of spring constant $k$.

Yielding in the SGR model is regarded not as a purely strain-induced phenomenon, but as an "activated" process. A mesoscopic element strained by an amount $l$ has a certain probability of yielding in a unit time interval. This rate is $\tau^{-1}$, where the characteristic yield time for the element with a yield energy $E=\frac{1}{2} k l_{y}^{2}$ is

$$
\tau=\tau_{0} \exp \left[\left(E-\frac{1}{2} k l^{2}\right) / x\right],
$$

where $\tau_{0}$ is the "attempt" time and $x$ is an activation factor, regarded as an effective "noise" temperature or, alternatively, as the typical energy available for the activated pro- 
cess. For $x<1$ the theoretical flow curve, i.e., a macroscopic stress response $\sigma(\dot{\gamma})$ to a steady shear rate $\dot{\gamma}$, has a macroscopic yield stress: $\sigma(\dot{\gamma} \rightarrow 0)=\sigma_{y}>0$. This behavior is characteristic of a glass phase. If such a system is strained below $\sigma_{y}$, it ages, while if $\sigma>\sigma_{y}$, the system achieves a steady state and aging no longer occurs. In the so-called "transient" regime, $1<x<2$ aging is absent and the behavior of the system is dominated by yielding of the mesoscopic elements.

There are two possibilities of yielding for the elements. If the element was strained up to the yield point, $1 / 2 k l^{2} \approx E$, it experiences a "strain-induced" yield event. On the other hand, an element with an energy much below the yield point can yield through activation dynamics once $E-1 / 2 k l^{2} \approx x$ (a "noise-induced" yield event).

An alternative description of the material elements used in the SGR model is the description of a particle moving in a landscape of quadratic potential wells of depth $E$. The bottom of each potential well corresponds to the unstrained state. Yielding is associated with the hopping of the particle to the bottom of a neighboring potential well.

The constitutive equations for the SGR model for a system prepared at time zero in an initial state of zero stress and strain and subjected to a time-dependent macroscopic strain $\gamma(t)(\gamma(t)=0$ for $t \leqslant 0)$ are [2]

$$
\begin{gathered}
\sigma(t)=\gamma(t) G_{0}(Z(t, 0))+\int_{0}^{t}[\gamma(t) \\
\left.-\gamma\left(t^{\prime}\right)\right] Y\left(t^{\prime}\right) G_{p}\left(Z\left(t, t^{\prime}\right)\right) d t^{\prime}, \\
1=G_{0}(Z(t, 0))+\int_{0}^{t} Y\left(t^{\prime}\right) G_{p}\left(Z\left(t, t^{\prime}\right)\right) d t^{\prime},
\end{gathered}
$$

with functions $Z\left(t, t^{\prime}\right), G_{p}(Z)$, and $G_{0}(Z)$ defined as

$$
\begin{gathered}
Z\left(t, t^{\prime}\right)=\int_{t^{\prime}}^{t} \exp \left(\left[\gamma\left(t^{\prime \prime}\right)-\gamma\left(t^{\prime}\right)\right]^{2} / 2 x\right) d t^{\prime \prime}, \\
G_{p}(Z)=\int_{0}^{\infty} \rho(E) \exp \left(-Z e^{-E / x}\right) d E, \\
G_{0}(Z)=\int_{0}^{\infty} P_{0}(E) \exp \left(-Z e^{-E / x}\right) d E .
\end{gathered}
$$

In these equations $Y\left(t^{\prime}\right)$ is the average yield rate at time $t^{\prime}$, $P_{0}(E)$, and $\rho(E)$ are correspondingly the probability distribution for the yield energies in the initial state at $t=0$ and in the consequent states, $t>0$. As shown in [1] the predictions of the SGR model are independent of the details of sample preparation and $P_{0}(E)$ could be taken equal to $\rho(E)$.

The same choice of units and of distribution of the yield energies as in [1] have been made: $\rho(E)$ $=\left(1 / x_{g}\right) \exp \left(-E / x_{g}\right)$ where the mean yield energy $x_{g}=\langle E\rangle$ is taken to be equal to unity. Consequently, with this choice of units $\rho(E)=\exp (-E)$.

An alternative form of the constitutive equations that we will use to calculate the relaxation modulus could be obtained by substitution of Eq. (7) into Eq. (6) [1] as

$$
\sigma(t)=\gamma(t)-\int_{0}^{t} \gamma\left(t^{\prime}\right) Y\left(t^{\prime}\right) G_{p}\left(Z\left(t, t^{\prime}\right)\right) d t^{\prime} .
$$

For the case described in our experimental procedure when the strain of the same amplitude is applied to the system after equal time intervals $\Delta t$, strain can be expressed as

$$
\begin{aligned}
\gamma(t)= & \gamma_{0} \theta(t)+\gamma_{0} \theta(t-\Delta t)+\gamma_{0} \theta(t-2 \Delta t)+\cdots \\
& +\gamma_{0} \theta(t-(n-1) \Delta t) \\
= & \sum_{j=0}^{n-1}(j+1) \gamma_{0} \theta(t-j \Delta t)
\end{aligned}
$$

with the function $\theta(t)=1$ for $t>0$ and zero elsewhere. Substitution of the $\gamma(t)$ function into Eq. (8) gives for the $n$th measurement $[t>(n-1) \Delta t], \quad n>1$ and $(j-1) \Delta t<t^{\prime}$ $<j \Delta t, \quad j=1, \ldots, n-1$,

$$
Z_{j}\left(t, t^{\prime}\right)=\alpha^{(n-j)^{2}}\left(t-t_{w}\right)+\Delta t\left(j+\sum_{i=0}^{n-j-1} \alpha^{i^{2}}\right)-t^{\prime},
$$

where $\alpha=\exp \left(\gamma_{0}^{2} / 2 x\right)$.

The relaxation modulus of the $n$th step could be found from the constitutive Eq. (11):

$$
\begin{aligned}
G(t- & \left.t_{w}, t_{w}, \gamma_{0}\right) \\
= & 1-\int_{0}^{t}\left[\frac{\gamma\left(t^{\prime}\right)}{n \gamma_{0}}\right] Y\left(t^{\prime}\right) G_{p}\left(Z\left(t, t^{\prime}\right)\right) d t^{\prime} \\
= & 1-\int_{0}^{t_{w}}\left[\frac{\gamma\left(t^{\prime}\right)}{n \gamma_{0}}\right] Y\left(t^{\prime}\right) G_{p}\left(Z\left(t, t^{\prime}\right)\right) d t^{\prime} \\
& -\int_{t_{w}=(n-1) \Delta t}^{t} Y\left(t^{\prime}\right) G_{p}\left(Z\left(t, t^{\prime}\right)\right) d t^{\prime} \\
= & 1-\sum_{j=1}^{n-1} \frac{j}{n} \int_{(j-1) \Delta t}^{j \Delta t} Y\left(t^{\prime}\right) G_{p}\left(Z_{j-1}\left(t, t^{\prime}\right)\right) d t^{\prime} \\
& -\int_{(n-1) \Delta t}^{t} Y\left(t^{\prime}\right) G_{p}\left(Z_{n-1}\left(t, t^{\prime}\right)\right) d t^{\prime} .
\end{aligned}
$$

To find an analytical expression for the relaxation modulus, the hopping rate has to be calculated. The expression for the hopping rate could be derived following the logic of the yielding rate calculation in the linear case [1]. By substituting $Z_{j}\left(t, t^{\prime}\right)$ into the second constitutive Eq. (7) and taking the Laplace transform of it, we derive the expression for the yielding rate for $x>1$ as

$$
\begin{aligned}
Y_{j}= & \frac{x-1}{x} \alpha^{(n-j)^{2}}+\frac{x-1}{x} \Gamma(x) \alpha^{(n-j)^{2}}\left[\alpha^{(n-j)^{2}}\left(t-t_{w}\right)\right. \\
& \left.+\Delta t\left(j+\sum_{i=0}^{n-j-1} \alpha^{i^{2}}\right)\right]^{1-x}+(x-1) \Gamma(x) \alpha^{1-x} t^{-x} \\
& +O\left(t^{2(1-x)}, t^{1-2 x}, \ldots\right)
\end{aligned}
$$


where $\Gamma$ is the gamma function.

Substituting this into Eq. (14) and performing the integration we have for $x>1$ and $t-t_{w} \ll t_{w}$

$$
\begin{aligned}
G\left(t-t_{w}, \Delta t, n, \gamma_{0}\right) \propto & \sum_{j=0}^{n-1} \frac{j}{n} \alpha^{(n-j)^{2}} \Gamma(x)\left(\alpha^{(n-j)^{2}}\left(t-t_{w}\right)\right. \\
& \left.+\Delta t \sum_{i=0}^{n-j-1} \alpha^{i^{2}}\right)^{1-x} \\
& +\Gamma(x) \alpha^{2-x}\left(t-t_{w}\right)^{1-x}
\end{aligned}
$$

The first term in Eq. (16) reflects the influence of all previous steps on the relaxation in the $n$th step. While the second term describes the relaxation as if there were no previous steps and a single large amplitude strain was applied to the system at time $t_{w}=(n-1) \Delta t$. In our experiments the residual stress is subtracted from $\sigma\left(t-t_{w}\right)$ at the beginning of each measurement. Consequently, the relaxation modulus for the $(n+1)$ th step will be expressed as

$$
\begin{aligned}
& G\left(t-t_{w}, \Delta t, n+1, \gamma_{0}\right) \\
& \propto \sum_{j=0}^{n} \frac{j}{n+1} \alpha^{(n+1-j)^{2}} \Gamma(x)\left(\alpha^{(n+1-j)^{2}}\left(t-t_{w}\right)\right. \\
& \left.\quad+\Delta t \sum_{i=0}^{n-j} \alpha^{i^{2}}\right)^{1-x}-\frac{n}{n+1} G\left(t=n \Delta t, \Delta t, n, \gamma_{0}\right) \\
& \quad+\Gamma(x) \alpha^{2-x}\left(t-t_{w}\right)^{1-x}
\end{aligned}
$$

or

$$
G\left(t-t_{w}, \Delta t, n, \gamma_{0}\right) \propto \Gamma(x) \alpha^{2-x}\left(t-t_{w}\right)^{1-x}+\delta
$$

with

$$
\begin{aligned}
\delta= & \Gamma(x)\left[\sum _ { j = 0 } ^ { n } \frac { j } { n + 1 } \alpha ^ { ( n + 1 - j ) ^ { 2 } } \left(\alpha^{(n+1-j)^{2}}(t-n \Delta t)\right.\right. \\
& \left.\left.+\Delta t \sum_{i=0}^{n-j} \alpha^{i^{2}}\right)^{1-x}\right]-\Gamma(x)(\Delta t)^{1-x}\left[\sum_{j=0}^{n-1} \frac{j}{n+1} \alpha^{(n-j)^{2}}\right. \\
& \left.\times\left(\sum_{i=0}^{n-j-1} \alpha^{i^{2}}\right)^{1-x}+\frac{n}{n+1} \alpha^{2-x}\right]
\end{aligned}
$$

An estimation of $|\delta|$ gives

$$
|\delta|<n^{2-x} \alpha^{n^{2}}(\Delta t)^{1-x} / 2 .
$$

Noting that Eq. (16) was derived in the short-time limit, i.e., $t-t_{w} \ll t_{w}$, and taking into account that $1-x<0$ we have $\left(t-t_{w}\right)^{1-x} \gg t_{w}^{1-x}$. Consequently, substituting for the $(n$ +1 ) th step $t_{w}$ by $n \Delta t$ and using the relation (20) we find that

$$
\left(t-t_{w}\right)^{1-x} \gg 2|\delta| /\left(n \alpha^{n^{2}}\right) .
$$

According to this relation the second term in Eq. (18) is small in comparison with the first term provided that the

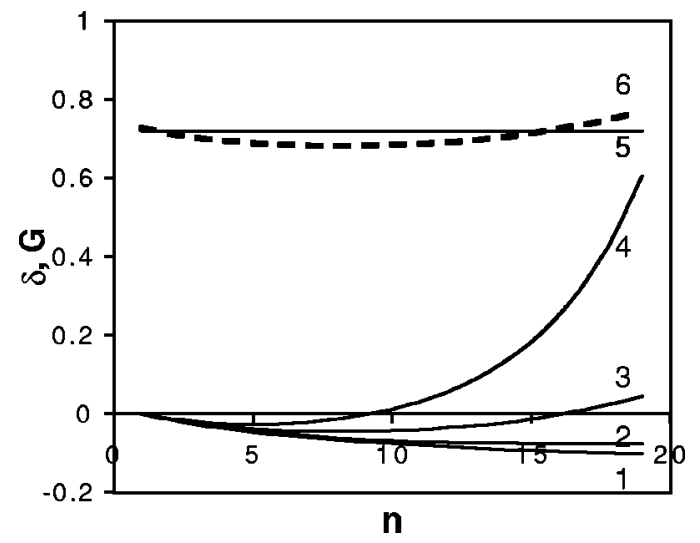

FIG. 2. Dependence of $\delta$ on the number of steps in strain for $x=1.2, t-t_{w}=5, \Delta t=300$, and $\gamma_{0}=0$ (curve 1), $\gamma_{0}=0.05$ (curve 2), $\gamma_{0}=0.1$ (curve 3), and $\gamma_{0}=0.15$ (curve 4). The main term and the resulting relaxation modulus are shown for $\gamma_{0}=0.1$ on curves 5 and 6 , respectively.

number of steps $n$ and the amplitude of the applied strain are not too high. In our case the system was subjected to strains of amplitude in the range of $0.03-0.15(20-100 \%)$ and the number of steps in strain was 19. Figure 2 illustrates the effect of the second term in Eq. (18) on the relaxation modulus. The term $\delta$ becomes comparable with the main term for the highest applied strain $(100 \%)$ and $n \geqslant 14$ only. In all other cases the approximation of the relaxation modulus with the main term in Eq. (18) is legitimate.

\section{RESULTS AND DISCUSSION}

\section{A. $20 \%$ strain}

It can be seen from Fig. 3 that all the relaxation curves except those where full relaxation is achieved, i.e., where $G$ drops to zero, are linear in a log-log plot. Thus, in these cases the relaxation modulus is a power function of the measuring time $G\left(t-t_{w}, t_{w}\right) \propto\left(t-t_{w}\right)^{\beta}$ with a negative power $\beta$. Examples of power-law fits of the relaxation curves are given in the figure. The deviation of the experimental data from the fits was found not to exceed $5 \%$.

Comparison of the experimental results with theoretical predictions shows that the experimental curves could be described by the function

$$
G\left(t-t_{w}, t_{w}\right) \propto\left(t-t_{w}\right)^{1-x}
$$

for the case $x>1$. Thus, in the following, the experimental results will be discussed in terms of the SGR model and in terms of the "noise temperature" $x$ associated with the probability of yielding rather than in terms of $\beta$. In terms of the model, stress relaxation proceeds through yield events, i.e., through the rearrangement of mesoscopic elements of the system into new configurations with zero stress. Consequently, the deviation from a power-law dependence of the relaxation modulus observed at some waiting times indicates yielding of most of the elements of the system. For these curves the slope of the first linear region following the hori- 


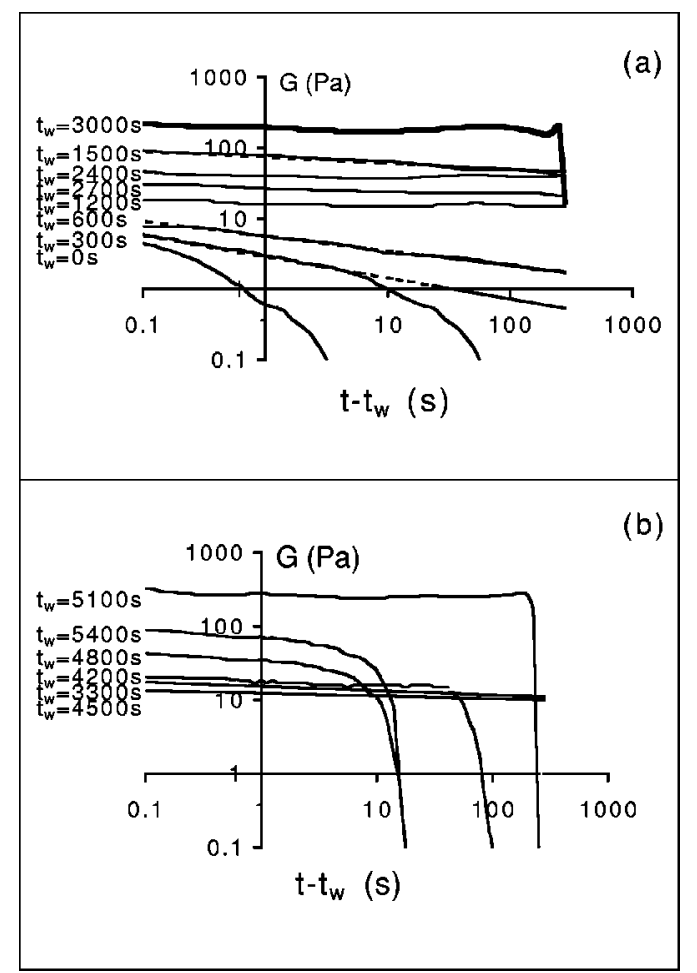

FIG. 3. Relaxation modulus of the SDS/pentanol/ cyclohexane/ $0.5 M$ brine system subjected to $20 \%$ strain vs the measuring time. The waiting time values $t_{w}$ at the beginning of each test are shown in the figure. For the sake of clarity not all of the experimental curves are shown. The dashed lines show fits to the data with a power law function $C\left(t-t_{w}\right)^{1-x}$.

zontal region, $t-t_{w}<0.1 \mathrm{~s}$ (not shown), in log-log plots was taken to calculate $x$ (Fig. 3, dashed lines).

The slope of the relaxation modulus varies from measurement to measurement, i.e., the $x$ value is a function of the waiting time. During the first two measurements the value of the noise temperature is high (Fig. 4) and the system experiences yielding, presumably associated with the orientation of domains with hexagonal symmetry in the shear field. The $x$ value decreases monotonically until it reaches a constant value. The decrease of the noise temperature and, consequently, the decreasing probability of yielding can be understood in the following way. After the yield energy is on average achieved, mesoscopic elements are able to hop to the bottom of neighboring potential wells with lower energy than the current one. The higher the number of yielded elements, the lower the average energy of the system. The lower average energy leads to a decrease in the probability of noiseinduced yield events, i.e., to a decrease in the $x$ value, as observed experimentally. Consequently, the noise temperature decreases until most of the mesoscopic elements have yielded and some new state with lower energy is reached.

It can be seen in Fig. 3 (for $t_{w} \leqslant 3000 \mathrm{~s}$ ) that the system does not fully relax during all measurements, except the first two. This means that the energy of the system does not return to the value characteristic of the unstrained state, but is accumulating in the system during the subsequent measurements. Consequently, the average energy of the mesoscopic
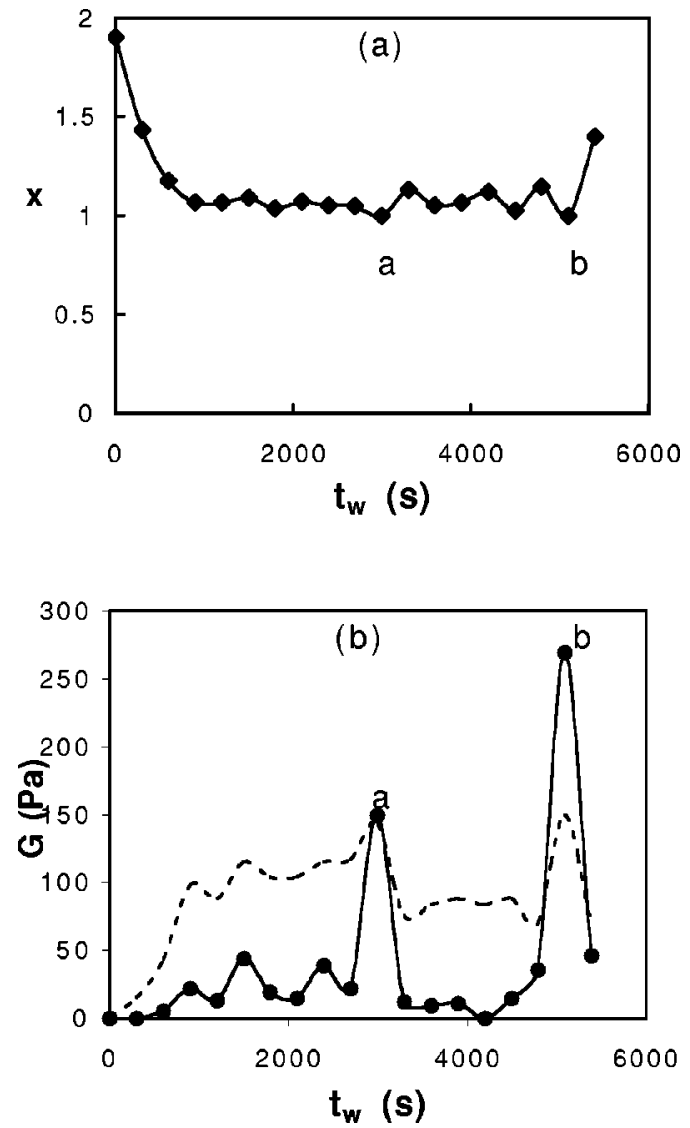

FIG. 4. Waiting time dependence of (a) noise temperature $x$ and (b) relaxation modulus $G\left(t-t_{w}=200 \mathrm{~s}, t_{w}\right)$ for the SDS/pentanol/cyclohexane/0.5M brine system subjected to $20 \%$ strain. The dashed line on the lower figure shows the function $150\left(t-t_{w}\right)^{1-x\left(t_{w}\right)}$ for $t-t_{w}=200 \mathrm{~s}$.

elements at the beginning of the relaxation measurements in the series $E\left(t=t_{w}\right), t_{w} \geqslant 600 \mathrm{~s}$, is an increasing function of the waiting time. It can be supposed that $E\left(t=t_{w}\right)$ will increase monotonically until it becomes close enough to the yield energy for the mesoscopic elements to hop to the bottom of a neighboring potential well. This transition is observed at a waiting time of $3250 \mathrm{~s}$ at the end of the 11th measurement (Fig. 3, upper graph, the thick curve). According to the SGR model, yielding of a mesoscopic element is associated with its rearrangement into a new equilibrium configuration with zero local strain. However, as shown in Fig. 2, the relaxation modulus, i.e., the average shear stress in the system $(G \propto \sigma)$, does not decrease to zero when yielding occurs during the 11th measurement, but becomes an order of magnitude lower than that before yielding. Consequently it is likely that only some mesoscopic elements have yielded at $t_{w}=3250 \mathrm{~s}$. If so, the average stress of the system

$$
\sigma=\frac{1}{N} \sum_{i=1}^{N} \sigma_{i}=\frac{1}{N} \sum_{i=1}^{n} \sigma_{i}+\frac{1}{N} \sum_{i=1}^{N-n} 0=\frac{1}{N} \sum_{i=1}^{n} \sigma_{i}
$$

where $N$ is the number of the mesoscopic elements in the system, $n$ is the number of elements that have not yielded, 
and $\sigma_{i}$ is the stress of the element $i$. Thus the higher the number of yielded elements the lower the resulting macroscopic stress of the system.

It can be supposed that two different states with different characteristic energies, presumably states with different orientations of the domains with hexagonal symmetry, coexist in the system after this yielding. The system remains in this transient state during the seven consequent measurements (waiting time interval 3250-5300 s) until the system experiences another yielding at the end of the 18th measurement. This time the stress in the system drops essentially to zero indicating that most of the mesoscopic elements have rearranged, adopting a new state.

The system in the transient state between the two yielding events is very unstable and experiences a number of weak yields before it adopts the new state of orientation. It is likely that the ratio of the number of mesoscopic elements in two states of orientation is changed via yielding. It should be noted that the $G$ value, i.e., the average stress of the mesoscopic elements, in the transient state is low (Fig. 3). Consequently the only way for yielding in this case is through activation dynamics, associated with interelement interactions. On the other hand at $t_{w}=3250 \mathrm{~s}$ and $5300 \mathrm{~s}$ the average energy of the system presumably comes close enough to the yield energy ( $G$ value is high) to cause strain-induced yield events to become more probable. The value of the noise temperature is equal to unity for these two measurements supporting the supposition that the transitions have a strain-induced character.

According to Eq. (18) the relaxation modulus, approximated by the first term, should be independent of the waiting time. However, as shown in Fig. 4(a) strong dependence of $G$ on the waiting time appears to be observed. This dependence cannot arise from the term $\delta$ in Eq. (18), which depends on the number of steps in strain, i.e., it is waiting time dependent. First of all this term is small compared to the main term in Eq. (18) and it has a waiting time dependence quite different from the experimental one (Figs. 2 and 4). The other possibility is that the waiting time dependence of $G$ is associated with the waiting time dependence of the noise temperature. As noted in [2], this is supposed to be a minimal extension to the SGR model, however the analysis of the SGR with $x$ evolving in time was not yet reported. On the base of our experimental data we propose to incorporate a waiting time dependence of $x$ into Eq. (18) not allowing $x$ to evolve with measuring time. Taking this into account a relatively good fit of the experimental curve was obtained with the function

$$
G\left(t-t_{w}, t_{w}\right) \propto\left(t-t_{w}\right)^{1-x\left(t_{w}\right)}
$$

(Fig. 4). Thus, according to this empirical relation, $G$ is independent of the waiting time when the noise temperature is constant and decreases or increases with $t_{w}$ according to the waiting time dependence of the $x$ value.

We propose a qualitative interpretation of the waiting time dependence of the relaxation modulus. First we assume a situation where the probability of yield events is low, i.e., when the energies of the mesoscopic elements are well be- low the yield energy and the $x$ value is low. In this case the average stress of the system will have the same increment at the beginning of each measurement and the $G$ value will be independent of the waiting time [the residual stress is subtracted from the $\sigma\left(t-t_{w}\right)$ at the beginning of each measurement].

If the energies of most of the mesoscopic elements are close to the yielding energy, but not close enough for the transition to take place during the current measurement, some of the mesoscopic elements may yield during the strain rise time of the subsequent measurement when an additional strain is applied to the system. This may change the $x$ value and lower the average stress of the system. Consequently, $G\left(t-t_{w}=0\right)$ will be lower than that at the beginning of the previous measurement. The energy distribution is expected to be bimodal in this case, i.e., one part of the elements will have low or zero energies and the other part will have energies close to the yielding value. The noise temperature is expected to increase relative to the value in the previous measurement reflecting the interactions between the yielded and unyielded elements. The higher the fraction of the yielded elements the higher the probability of yielding for the rest of the elements, and the higher the $x$ value.

Finally, we need to interpret our observation that the increase of the relaxation modulus is accompanied by a small drop in the noise temperature [Fig. 4, points $a$ and $b$ on the $G\left(t_{w}\right)$ curve correspond to points $a$ and $b$ on the $x\left(t_{w}\right)$ curve, respectively]. The low $x$ value suggests that the energy distribution of the elements during this measurement (we will denote this measurement by its number $n$ ) is narrow, i.e., the energies of the elements are close to the average value. Noting that the $x$ value is always relatively high in the previous measurement (the measurement number $n-1$ ), we suppose that the transition from the broad to narrow energy distribution takes place during the $(n-1)$ th measurement. Provided that no structural rearrangements were observed in the $(n$ $-1)$ th measurement, the average energy of the system at the end of the $(n-1)$ th measurement is close to, but below the yield energy. Consequently, at the beginning of the $n$th measurement the mesoscopic elements of the system receive an increment in strain, i.e., an increment in energy, such that the average energy of the system may become close enough to the yielding value for a strain-induced transition to take place. The difference between the situation when the distribution at the end of the $(n-1)$ th measurement is broad or narrow is the following. If the system with the broad distribution of energies of mesoscopic elements is strained, the elements with high energies, i.e., with the energies close to the yielding value, can yield thereby acquiring zero strain. As was pointed out above, this will lower the average energy of the system. On the other hand, if the energy distribution is narrow no yielding will occur during the strain rise time and the average stress of the system at $t-t_{w}=0$ will be higher than that in the previous case.

Thus the increase in $G\left(t-t_{w}=0\right)$ is presumably associated with the mechanism of the strain-induced transitions, while the decrease in this value is due to the noise-induced yield events. 

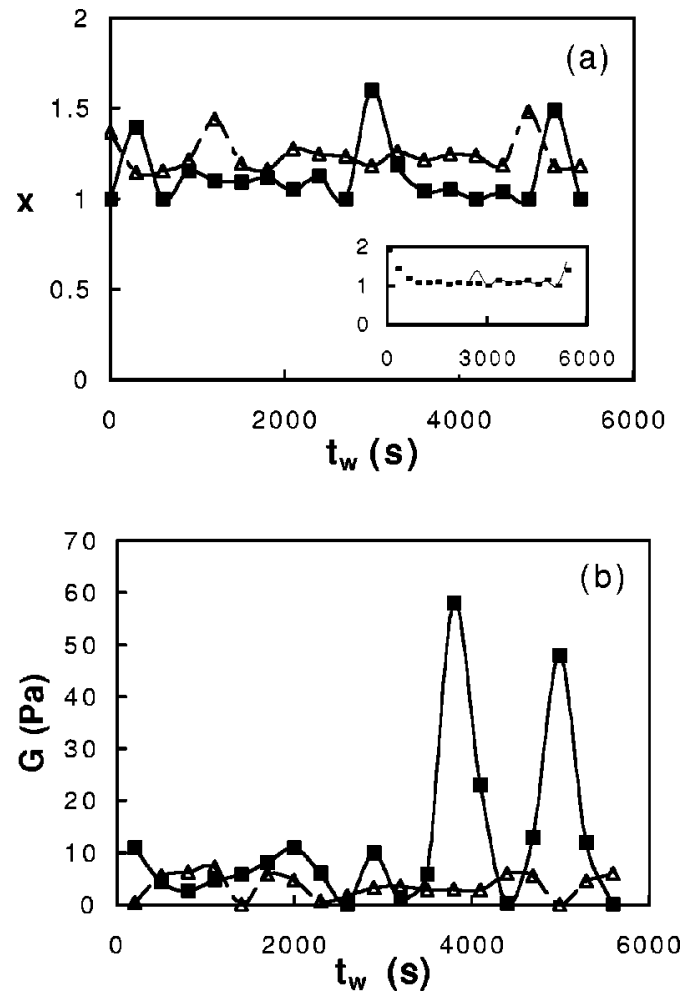

FIG. 5. Waiting time dependence of (a) noise temperature $x$ and (b) relaxation modulus $G\left(t-t_{w}=200 \mathrm{~s}, t_{w}\right)$ for the SDS/pentanol/cyclohexane/0.5M brine system subjected to $40 \%$ strain (squares) and 60\% strain (triangles). The inset in (a) shows the waiting time dependence of $x$ shifted by $2400 \mathrm{~s}$ along the $t_{w}$ axis for the system subjected to $40 \%$ strain (solid line) and $x\left(t_{w}\right)$ for the same system subjected to $20 \%$ strain (dashed line).

\section{B. 40-100\% strain}

The profile (variation with $t_{w}$ ) and the value of the noise temperature both depend on the applied strain. For the series of relaxation measurements with an applied strain of $40 \%$, the noise temperature and the probability of yielding are high. When $40 \%$ strain is applied to the system, the noise temperature exhibits a maximum during the second measurement in the series (Fig. 4). An abrupt drop in the noise temperature is observed in the next measurement, presumably due to yielding of most of the mesoscopic elements of the system. After this drop in the $x$ value, the profile of the noise temperature dependence on the waiting time in the region $600 \leqslant t_{w} \leqslant 3000 \mathrm{~s}$ repeats that for the previous series with an applied strain of $20 \%$ in the region between the two transitions, i.e., for $3000 \leqslant t_{w} \leqslant 5400 \mathrm{~s}$ [inset in Fig. 5(a)]. This indicates that when higher strain is applied the first transition takes place during the third measurement in the series instead of in the 11th in the previous case. In other words the energy required for most of the mesoscopic elements to rearrange themselves into a new state is available almost immediately (note that it is a strain-induced transition). Conversely when lower strain is applied, the system has to collect the energy during a number of measurements until it is sufficient for the transition to take place. After the second transition, the system submitted to $40 \%$ strain undergoes a number of strainand noise-induced transitions.
Structural data are required to detect the orientation of the domains in the system in the shear field and they will be reported in a future publication. However, according to the rheo-small angle light scattering data of Richtering et al. [10], a surfactant hexagonal phase subjected to constant shear stress undergoes transitions from an isotropic distribution of the domains, to a perpendicular orientation, and then through a regime of coexistence of parallel and perpendicular orientations to the formation of a monodomain with the director in the flow direction. It can be supposed that in the case of applied strain, our system undergoes similar transitions. Consequently, the first transition may be associated with the orientation of the domains in the perpendicular orientation. The second-with the rearrangement of some of the domains parallel to the flow direction-and the subsequent transitions may be associated with an increase of the fraction of parallel-oriented domains and then with monodomain formation.

For the $60 \%$ strain experiment the average $x$ value is higher than that obtained at $40 \%$ strain and fluctuates between $x=1.2$ and 1.5, indicating that the system is in a transient state (Fig. 5). Presumably the external strain is high enough for the system to adopt the same state as in the $40 \%$ strain experiment after the second transition, i.e., the state of coexistence of domains perpendicular and parallel to the flow orientations. On the other hand, the $60 \%$ applied strain might not be sufficient to induce monodomain formation. However, it is likely that a monodomain is formed during the series of experiments with $80 \%$ applied strain (Fig. 6). In this case after yielding during the first measurement in the series the noise temperature is almost constant and equal to 1.2, that is close to the $x$ value for the case of the $60 \%$ applied strain. An abrupt increase in the $x$ value observed at $t_{w}$ $=1500-1800 \mathrm{~s}$ is followed by a region of nearly constant noise temperature. The noise temperature is close to unity in this region, indicating that the system has reached a steady state with a higher degree of order where the probability of noise-induced yield events is very low. It can be supposed that from close to the beginning of the series most of the domains are aligned in the flow direction. The size of these domains might increase due to interdomain interactions until a monodomain is formed at $t_{w} \approx 2000 \mathrm{~s}$.

The profile of the noise temperature dependence on the waiting time in the case of the $100 \%$ applied strain is similar to that in the previous case (Fig. 6). The only difference between these two cases is that the transition is shifted on the waiting time scale to $t_{w}=900 \mathrm{~s}$ and is narrower than in the previous case.

The value of the relaxation modulus depends on the waiting time, in agreement with the above qualitative picture. For all cases except the case of the first transition in the system subjected to $20 \%$ strain the $G$ value drops to zero when the transitions take place, indicating that most of the mesoscopic elements have yielded to the unstrained state. The average stress values when $60 \%$ strain is applied are the same as those in the cases of $80 \%$ and $100 \%$ applied strain, before the transition, indicating that the system is in the same state.

However, this is not the case for the strain-induced transitions. It can be seen from Fig. 5 that the relaxation modulus 

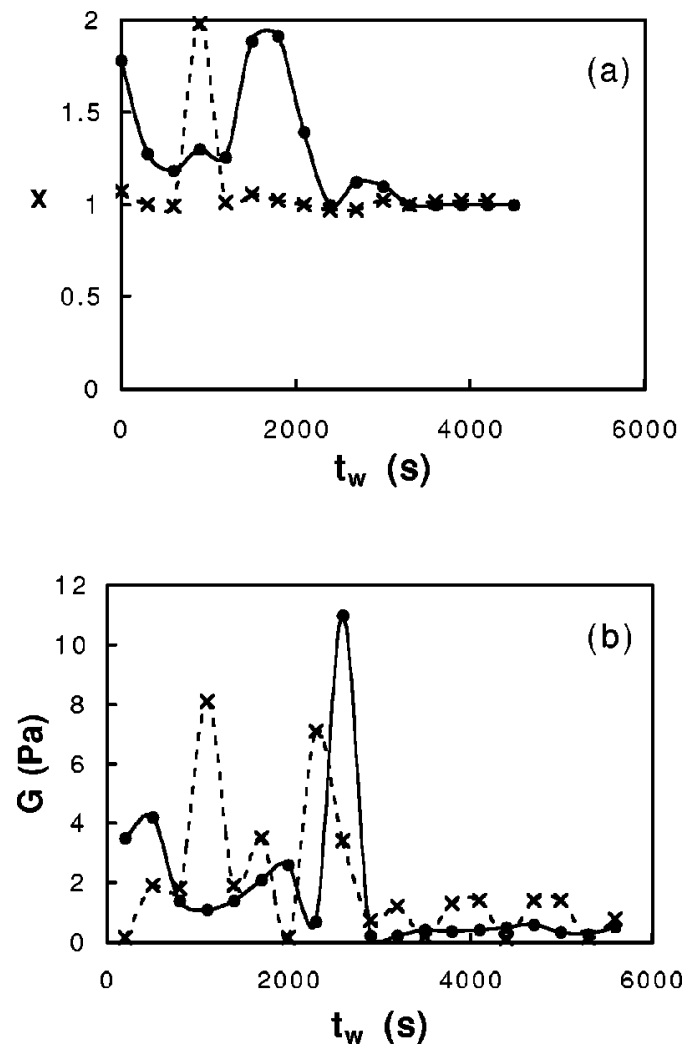

FIG. 6. Waiting time dependence of (a) noise temperature $x$ and (b) relaxation modulus $G\left(t-t_{w}=200 \mathrm{~s}, t_{w}\right)$ for the SDS/pentanol/cyclohexane/ $0.5 M$ brine system subjected to $80 \%$ strain (circles) and 100\% strain (crosses).

obtained in the experiments with $40 \%$ applied strain is more than an order of magnitude lower than that in $20 \%$ strain experiment in the region around the first two transitions. This result is the manifestation of the speedup of the relaxation process in the nonlinear regime. As shown in [2], a large single step strain $\gamma_{0}$ speeds up the relaxation by a factor $\exp \left(\gamma_{0}^{2} / 2 x\right)$. In the single step strain approximation this result may be applied to each test in our series. Thus the higher the applied strain the higher the speedup of relaxation. This effect explains also the shift of the transition times on the waiting time scale to lower waiting times with $\gamma_{0}$.

\section{Effect of composition}

Investigations of the relaxation in the SDS/pentanol/ cyclohexane/brine system in lower ionic strength brine with a slightly smaller spacing between cylinders [17], revealed that the system experiences similar transitions as in the higher ionic strength case. However, the transitions induced by low strain, i.e., the transitions presumably associated with the rearrangement of domains, are shifted on the waiting time scale to shorter waiting times. For example, the transitions, induced by $20 \%$ strain, are shifted by $3000 \mathrm{~s}$ towards shorter waiting times. However, a decrease in the value of the applied strain results in a decrease in the transition time shift. A linear dependence of the shift of transition time on applied strain was observed (Fig. 7). Thus a $19.3 \%$ strain was found to produce exactly the same effect on the $0.4 M$

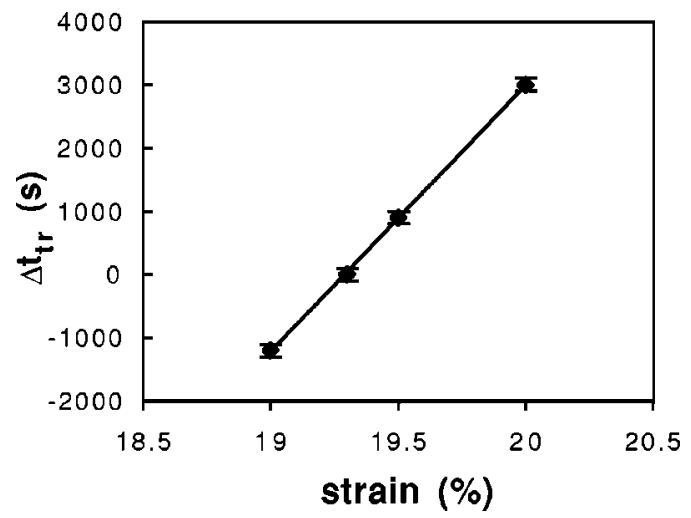

FIG. 7. Strain dependence of the transition time shift in the SDS/pentanol/cyclohexane/brine system with $0.4 M$ brine with respect to the transition times in SDS/pentanol/cyclohexane/brine system with $0.5 M$ brine subjected to $20 \%$ strain.

system as a $20 \%$ strain on the $0.5 M$ system. It should be noted that when $19 \%$ strain is applied no transitions except the first one at a waiting time of $4200 \mathrm{~s}$ were observed, because the subsequent transition is expected to occur $1500 \mathrm{~s}$ after the first one, i.e., at a waiting time of $5700 \mathrm{~s}$, which was not experimentally accessible.

Stronger electrostatic interactions in the system with the lower ionic strength of brine may be responsible for the observed shift in the transition times. Provided that in both systems the weight ratio of brine/SDS is the same, the screening of the SDS charge is different: the number of the excess counterions per SDS charge is 0.35 for the $0.5 M$ system (the Debye length is $0.4 \mathrm{~nm}$ ) and 0.28 for the $0.4 M$ system (the Debye length is $0.5 \mathrm{~nm}$ ). Consequently, the electrostatic repulsion between the cylinders is higher in the system with $0.4 M$ brine. This suggests a higher cooperativity for domain rearrangement. The stronger correlation between the elements suggests also that less strain is required to produce the same transitions. These considerations are supported by the fact that the noise temperature, reflecting the degree of interdomain interactions, is higher for the $0.4 \mathrm{M}$ system before the transitions take place, while the drop in the $x$ value after the transitions is steeper (Fig. 8). The noise temperature value oscillates between 1 and 1.27 in the region between the transitions, while in the $0.5 M$ system this value is almost constant and equal to 1.12. This means that when stronger intercylinder, and, consequently, interdomain interactions are present, more domains rearrange themselves into a new state simultaneously, causing a steeper drop in $x$ value.

The above considerations relate to the transitions associated with domain rearrangements only, but not to those associated with monodomain formation. Contrary to the low strain case, positive shifts in transition times are observed when the $0.4 M$ system was submitted to high strain. For example, monodomain formation presumably takes place at $t_{w}=900 \mathrm{~s}$ in the $0.5 \mathrm{M}$ system subjected to $100 \%$ strain, while under the same experimental conditions the $0.4 M$ system experiences such a transition $3300 \mathrm{~s}$ later, at $t_{w}$ $=4200 \mathrm{~s}$ (Fig. 9).

As in the case of the low applied strain the shift in the transition time is equivalent to the shift in the applied strain 


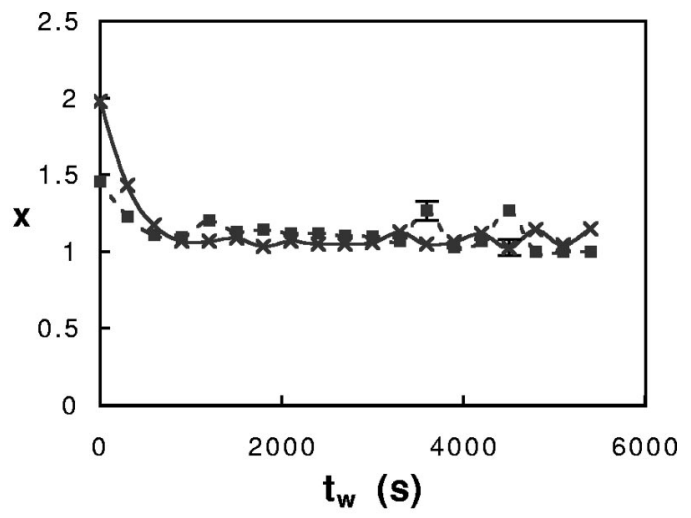

FIG. 8. Noise temperature dependence on waiting time for the SDS/pentanol/cyclohexane/brine system with $0.5 M$ brine subjected to $20 \%$ strain (crosses) and with $0.4 M$ brine subjected to $19.3 \%$ strain (squares).

value. The waiting time dependence of the relaxation modulus for the $0.4 M$ sample with $80 \%$ applied strain is similar in profile to that for the $0.5 \mathrm{M}$ system with a $60 \%$ applied strain (Fig. 10). The lowest strain that (presumably) induces monodomain formation in the $0.4 M$ system was found to be equal to $83.3 \%$ (Fig. 11), while for the $0.5 M$ system $80 \%$ strain induces such a transition in the middle of the series (Fig. 6). Thus, on the applied strain scale the transitions associated with monodomain formation are shifted towards higher strain. A linear dependence of the transition time shift on the applied strain was also observed (Fig. 9). Again we attempt to interpret these results from the point of view of the intercylinder, interdomain interactions.

In the case of the low applied strain, stronger interdomain repulsion in the $0.4 M$ system induces stronger element (domain) coupling, because the rearrangement of a domain may lead to a decrease in the distances between the cylinders of this domain and those of the neighboring ones. This induces an increase in the electrostatic repulsion between the yielded domain and the neighboring ones and, consequently, leads to an increase in the probability of the neighboring domain rearranging. Provided that the Debye lengths in $0.5 \mathrm{M}$ and

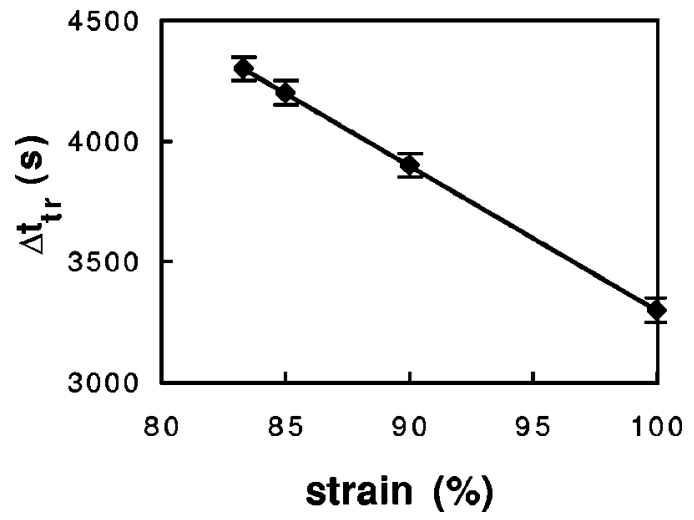

FIG. 9. Strain dependence of the transition time shift in the SDS/pentanol/cyclohexane/brine system with $0.4 M$ brine with respect to the transition times in the same system with $0.5 \mathrm{M}$ brine subjected to $100 \%$ strain.

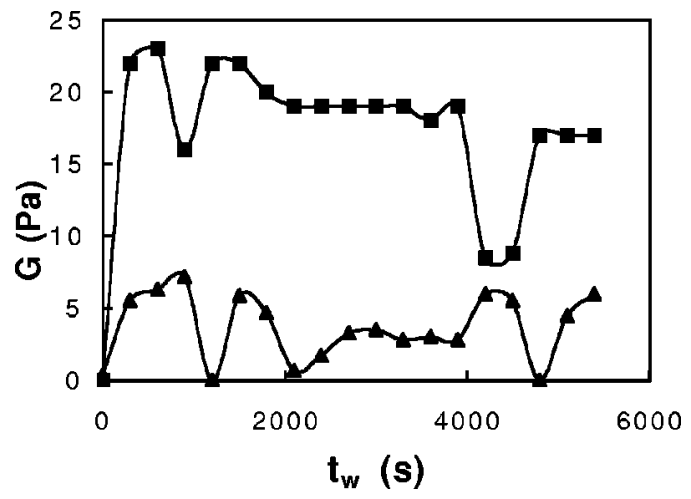

FIG. 10. Waiting time dependence of the relaxation modulus for the SDS/pentanol/cyclohexane/brine system with $0.5 M$ brine submitted to $60 \%$ strain (triangles) and with $0.4 M$ brine subjected to $80 \%$ strain (squares).

$0.4 M$ systems do not differ much, the shift on the applied strain scale required to induce the transitions in both systems at the same waiting times is low, $\Delta \gamma=0.7 \%$. However, $\Delta \gamma$ is higher than $20 \%$ in the case of the high applied strain, i.e., when most of the domains presumably have the same orientation and the straining of the system induces a monodomain formation. Monodomain formation implies the annihilation of domain boundaries due to cylinder fusion. Consequently, in this case electrostatic repulsion along the cylinders rather than much weaker intercylinder repulsion plays the main role. Thus, the higher the repulsion along the cylinder is, i.e.,

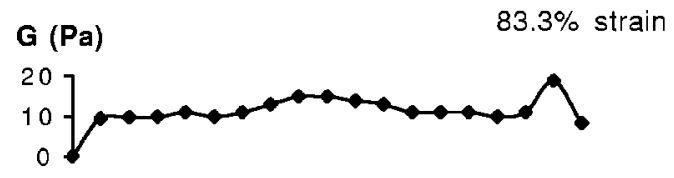

$85 \%$ strain

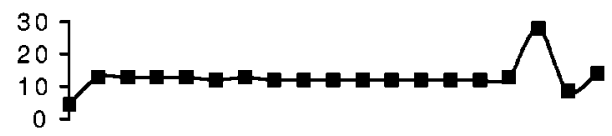

$90 \%$ strain
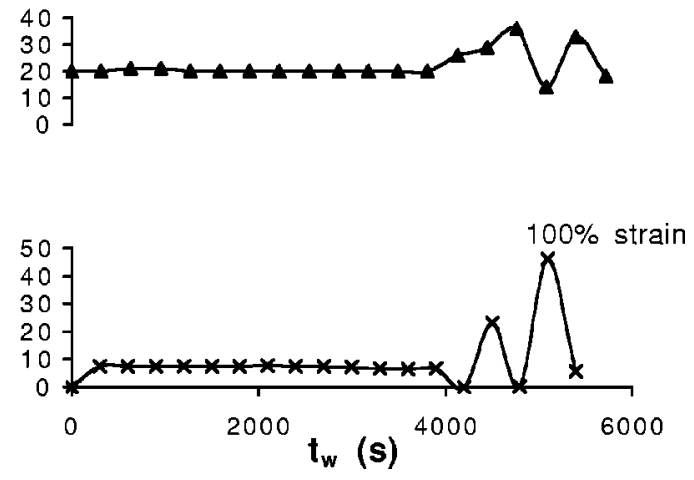

FIG. 11. Waiting time dependence of the relaxation modulus for the SDS/pentanol/cyclohexane/ $0.4 M$ brine system subjected to $83.3 \%, 85 \%, 90 \%$, and $100 \%$ strain. 
the lower the SDS charge screening, the higher the strain that should be applied to induce monodomain formation.

\section{CONCLUSION}

An expression for the relaxation modulus for the case of the $n$ steps of equal strain amplitude and equal length applied to a sample in the transient regime has been derived within the framework of the SGR model. The experimental data on relaxation in the SDS/pentanol/cyclohexane/brine system in this case showed a more complicated behavior of the relaxation modulus than proposed in the theory. However, allowing the noise temperature to evolve with the waiting time, we found a qualitative agreement between the calculated and the experimental data.

Depending on the amplitude of the applied strain and the

[1] S. M. Fielding, P. Sollich, and M. E. Cates, J. Rheol. 44, 323 (2000).

[2] P. Sollich, Phys. Rev. E 58, 738 (1998).

[3] T. G. Mason, J. Bibette, and D. A. Weitz, Phys. Rev. Lett. 75, 2051 (1995).

[4] S. A. Khan, C. A. Schnepper, and R. C. Armstrong, J. Rheol. 32, 69 (1988).

[5] P. Panizza et al., Langmuir 12, 248 (1996).

[6] M. Cloitre, R. Borrega, and L. Leibler, Phys. Rev. Lett. 85, 4819 (2000).

[7] E. van der Aershot and J. Mewis, Colloids Surface 69, 15 (1992).

[8] D. Bonn et al., Europhys. Lett. 45, 52 (1998).

[9] M. Lukaschek, D. A. Grabowski, and C. Schmidt, Langmuir 11, 3590 (1995); I. Koltover et al., J. Phys. II 6, 893 (1996); C. E. Fairhurst, M. C. Holmes, and M. S. Leaver, Langmuir 12, 6336 (1996); G. Schmidt, W. Richtering, P. Linder, and P. Al- number of steps in strain, the SDS/pentanol/cyclohexane/ brine system was found to experience a number of strainand noise-induced transitions. The transitions in the system are presumably associated with the rearrangement of the domains having two-dimensional hexagonal symmetry when the applied strain is low, and with monodomain formation when a high strain is applied to the system. However, simultaneous rheological and $\mathrm{x}$-ray investigations are required to prove this supposition, and these experiments are currently in progress.

\section{ACKNOWLEDGMENTS}

This work was funded by EPSRC Grant No. GR/ M50850. We thank Professor P.E. Luckham for the generous gift of the Bohlin rheometer.

exandridis, Macromolecules 31, 2293 (1998).

[10] W. Richtering, J. Lauger, and R. Linemann, Langmuir 10, 4374 (1994).

[11] R. G. Larson and H. C. Ottinger, Macromolecules 24, 85 (1996).

[12] S. Muller, P. Fischer, and C. Schmidt, J. Phys. II 7, 421 (1997).

[13] G. Schmidt et al., J. Chem. Phys. 102, 507 (1998).

[14] F. Morisson et al., Macromolecules 26, 5271 (1993).

[15] C. Jackson et al., Macromolecules 28, 713 (1995).

[16] G. Schmidt, S. Muller, C. Schmidt, and W. Richtering, Rheol. Acta 38, 486 (1999).

[17] L. Ramos and P. Fabre, Langmuir 13, 682 (1997).

[18] L. Ramos and F. Molino, Europhys. Lett. 51, 320 (2000).

[19] Mechanical Behavior of Materials, edited by F. A. McClintock and A. S. Argon (Addison-Wesley, Reading, MA, 1966), pp. $626,637$. 